\section{A. D’Urzo}

Family and Community Medicine, University of Toronto, Toronto, Canada.

Correspondence: A. D’Urzo, Family and Community Medicine, University of Toronto, 1670 Dufferin Street, Suite, 107, Toronto, Ontario M6H 3M2, Canada. E-mail: tonydurzo@sympatico.ca

Statement of Interest: A statement of interest for A. D'Urzo can be found at www.erj.ersjournals.com/site/misc/statements.xhtml

\section{REFERENCES}

1 Luks VP, Vandemheen KL, Aaron SD. Confirmation of asthma in an era of over diagnosis. Eur Respir J 2010; 36: 255-260.

DOI: $10.1183 / 09031936.00136210$

\section{To the Editors:}

The study by LUKS et al. [1] published in the August 2010 issue of the European Respiratory Journal (ERJ) evaluates a diagnostic algorithm to confirm previous physician-diagnosed asthma, and concludes that pre- and post-bronchodilator spirometry or a single methacholine challenge test (MCT) is sufficient to confirm the diagnosis in $>90 \%$ of patients. In the remaining patients, a repeat MCT after partial or complete drug withdrawal is confirmatory. The study was prompted by the epidemiological trends showing increasing prevalence of asthma, raising the question of a real increase versus an overdiagnosis. Correct diagnosis is extremely important issue, as a correct estimate of the burden of disease has major medical, social and economic implications. The study has also received editorial comments by CONTOLI and PAPI [2] in the same issue of the ERJ.

There is an inherent assumption in the study that pre- and post-bronchodilator spirometry or MCT are gold standards for diagnosis of asthma, and those cases failing these tests can be labeled as "overdiagnosed". The criteria of an improvement in forced expiratory volume in $1 \mathrm{~s}$ (FEV1) of $\geqslant 200 \mathrm{~mL}$ and $\geqslant 15 \%$ after bronchodilator, used by the authors, lacks consensus. In fact, there are several debatable issues regarding the test of bronchodilator responsiveness, which is the best parameter to assess the response (forced vital capacity (FVC) or FEV1); what is a positive threshold of change $(12,15$ or $20 \%$ ) and what is the best method of expressing the result (percentage change over baseline or change as a percentage of predicted) [3]? The recommendations have varied from time to time and from country to country, and the recent consensus statement of the joint American Thoracic Society (ATS)/European Respiratory Society (ERS) task force considers an increase in FEV1 or FVC of $12 \%$ and $200 \mathrm{~mL}$ over the baseline as an indicator of responsiveness [4]. We have previously shown that bronchodilator responsiveness has limited application as a gold standard diagnostic test for asthma, as none of the above methods of expression of the response have adequate positive predictive value [5]. Patients with chronic obstructive pulmonary disease (COPD) are likely to meet the aforementioned criteria in nearly $20 \%$ of cases.

However, an MCT also has deficiencies as a confirmatory test. A provocative concentration of methacholine causing a $20 \%$ fall in FEV1 of $<8 \mathrm{~g} \cdot \mathrm{mL}^{-1}$ lacks adequate specificity [2] and false positive results are often seen in patients with other diseases, including COPD, allergic rhinitis and sarcoidosis. In their editorial comment on the article, CONTOLI and PAPI [2] have referred to limitations in its sensitivity and specificity that result in both false positive and negative results. This undermines its value as a diagnostic test.

The basic premise of the study is thus flawed and the conclusions based on this assumption would also be erroneous. Both the tests introduce a substantial dimension of over- and underdiagnosis and, hence, are not appropriate tools to evaluate a previous diagnosis.

Overdiagnosis may result from a lack of availability or choice of diagnostic methods, or an incorrect application and interpretation of these. The title of the article refers to "an era of overdiagnosis". However, in the evolution of epidemiological trends in asthma, the contribution of underdiagnosis is equally important. Several studies have documented the phenomenon of the iceberg of asthma morbidity pointing towards substantial underdiagnosis in asthma, which is also often diagnosed late in its course [6,7]. The study by LUKS et al. [1] was not designed to look into the underdiagnosis of asthma. An "era of misdiagnosis" may be a more apt phrase for the title than "an era of overdiagnosis".

While appreciating the study by LUKS et al. [1], CONTOLI and PAPI [2] have remarked that the suggested algorithm to reduce overdiagnosis of asthma is valuable but perfectible, and needs to be improved in order to better identify truly negative results. We believe that in the absence of a gold standard for diagnosis, a weighted, multidimensional diagnostic scoring system using multiple tools, including history, therapeutic responsiveness to drugs and objective tests, such as pre- and post-bronchodilator spirometry, MCT or peak flow variability, needs to be developed that can be adapted and applied at all levels of healthcare, from primary to tertiary. This would allow an evaluation of both over- and underdiagnosis in the epidemiological trends. We are still short of attaining perfection in the diagnosis of asthma in epidemiological studies or in clinical practice.

\section{S.K. Chhabra and M. Gupta}

Viswanathan Chest Hospital, Vallabhbhai Patel Chest Institute, University of Delhi, Delhi, India.

Correspondence: S.K. Chhabra, Department of Cardiorespiratory Physiology, Viswanathan Chest Hospital, Vallabhbhai Patel Chest Institute, University of Delhi, Delhi 110007, India. E-mail: skchhabra@mailcity.com

Statement of Interest: None declared. 


\section{REFERENCES}

1 Luks VP, Vandemheen KL, Aaron SD. Confirmation of asthma in an era of overdiagnosis. Eur Respir J 2010; 36: 255-260.

2 Contoli M, Papi A. When asthma diagnosis becomes a challenge Eur Respir J 2010; 36: 231-233.

3 Chhabra SK, Vijayan VK, Gupta RK, et al. Expression of bronchodilator response: comparison of four indices. Respir Medicine 2002; 96: 611-614.

4 Pellegrino R, Viegi G, Brusasco V, et al. Interpretative strategies for lung function tests. Eur Respir J 2005; 26: 948-968.

5 Chhabra SK. Acute bronchodilator response has limited value in differentiating bronchial asthma from COPD. J Asthma 2005; 42 : 367-372.

6 van Schayck CP, van der Heijden FMMA, van den Boom G, et al. Underdiagnosis of asthma: is the doctor or the patient to blame? The DIMCA project. Thorax 2000; 55: 562-565.

7 Enright PL, McClelland RL, Newman AB, et al. Underdiagnosis and undertreatment of asthma in elderly. Chest 1999; 116: 603-613.

DOI: $10.1183 / 09031936.00140910$

\section{From the authors:}

A. D’Urzo has correctly identified from our data [1] that bronchial challenge testing seems to be a more sensitive test to confirm asthma compared to pre- and post-bronchodilator spirometry. Our results were similar to those of GOLDSTEIN et al. [2] who demonstrated that bronchial challenge testing with methacholine has far greater sensitivity to diagnose asthma compared with post-bronchodilator spirometry. Despite these findings, pre- and post-bronchodilator spirometry should probably be the first-line test to diagnose or confirm asthma for several reasons. Inducing bronchoconstriction with a bronchial challenge test poses a risk to the patient that could be avoided if that patient is able to have asthma diagnosed, or confirmed, with post-bronchodilator spirometry. Admittedly, the risk may be more theoretical than evidence-based. For example, to date there are no reported deaths from methacholine challenge testing [3] and there are studies demonstrating that it is quite safe, even in patients with severe obstruction [4]. That being said, there have been fatalities following specific antigen challenges and the current American Thoracic Society guidelines cite moderate obstruction (forced expiratory volume in $1 \mathrm{~s}$ (FEV1) $\leqslant 60-70 \%$ predicted) as a contraindication to methacholine challenge testing [3]. Bronchial challenge testing is also more time consuming and more expensive than spirometry, less readily available than spirometry and potentially uncomfortable for patients (approximately a third of patients develop symptoms) [3]. Furthermore, a physician must be available onsite during the bronchial challenge test, which adds to expense. In contrast to bronchial challenge testing, pre- and post-bronchodilator spirometry is completely safe, inexpensive and can be more easily performed in primary care. Thus, although sensitivity is an issue, we feel that preand post-bronchodilator spirometry, which is the cheapest, safest and most readily available test, is probably preferred as a first step in confirming a diagnosis of asthma.

S.K. Chhabra and M. Gupta correctly state that there is debate and a relative lack of gold standard criteria on which to base a diagnosis of asthma. We used first-step criteria of FEV1 improvement $\geqslant 200 \mathrm{~mL}$ and $\geqslant 15 \%$ (rather than $12 \%$ ) because, as S.K. Chhabra and M. Gupta point out, bronchodilator responsiveness lacks specificity. Our intention was to be conservative and to avoid false-positive results in the first step of the diagnostic algorithm. As all patients who did not exhibit $15 \%$ reversibility proceeded to a bronchial challenge test we surmised that true asthmatics would have their diagnosis confirmed at the following visit. S.K. Chhabra and M. Gupta also point out that bronchodilator responsiveness lacks specificity and can be positive in patients with chronic obstructive pulmonary disease (COPD). We agree that many patients with COPD may exhibit bronchodilator responsiveness and positive bronchial challenge tests, therefore, our study excluded any patients with a $>10$ pack-yr smoking history, specifically to avoid applying our diagnostic algorithm to patients with possible COPD.

The aim of our study was to confirm a diagnosis of asthma in those who had already received a physician diagnosis of asthma in the past. According to the Canadian Asthma Consensus guidelines, asthma is defined as "A disease characterised by paroxysmal or persistent symptoms of dyspnoea, chest tightness, wheezing, sputum production and cough, associated with variable airflow limitation and airway hyperresponsiveness to endogenous or exogenous stimuli" [5]. Similarly the Global Initiative for Asthma (GINA) guidelines define asthma as "a chronic inflammatory disorder of the airways associated with airway hyperresponsiveness that leads to recurrent episodes of wheezing, breathlessness, chest tightness and coughing, particularly at night or in the early morning" [6]. A necessary condition of both the Canadian and GINA definitions is that asthma must be diagnosed based on the evidence of: 1) respiratory symptoms; and 2) physiological evidence of reversible airflow obstruction and/or airway hyperresponsiveness. Thus, we used our algorithm to confirm physiological evidence of asthma in those who had been previously diagnosed. We agree with S.K. Chhabra and M. Gupta that in the absence of a gold standard for diagnosis, a weighted multi-dimensional diagnostic scoring system that incorporates physiological testing, patient history and symptoms, and perhaps responsiveness to asthma medications, needs to be developed to comprehensively evaluate patients for a diagnosis of asthma. To date, this 'gold standard' diagnostic scoring system remains elusive; however, we feel that studies such as ours are the first necessary step towards the development of such a gold standard.

\section{S. Aaron and V. Luks \\ Ottawa Health Research Institute, University of Ottawa, Ottawa, ON, Canada.}

Correspondence: S. Aaron, Ottawa Health Research Institute, University of Ottawa, Room 1812F, Ottawa Hospital, 201 Smyth Road, Ottawa K1 8L6, Canada. E-mail: saaron@ottawa hospital.on.ca

Statement of Interest: None declared. 